\title{
ALGUNAS CARACTERISTICAS DE LA POBLACION ESPAÑOLA SEGUN EL CENSO DE 1970
}

$310: 312$

por

Ignacio Ballester Ros

SUMARIO: I. INTRODUCCION.-II. LA COMPOSICION DE LA FAMILIA ESPAÑOLA.-III. LAS CARACTERISTICAS PERSONALES: EL SEXO, EL ESTADO CIVIL, LA EDAD Y LA NACIONALIDAD.-IV. EL LUGAR DE PROCEDENCIA DE LA POBLACION RESIDENTE.V. EL ANALFABETISMO Y EL NIVEL DE INSTRUCCION DE LOS habitaNTES: 1. El analfabetismo. 2. Niveles de estudio alcaNZADOS POR LA POBLACION OUE NO CURSABA ESTUDIOS EN EL MOMENTO CENSAL. 3. Niveles de estudio DE LOS ESTUdiantes EN EL MOMENTO CENSAL.

\section{INTRODUCCION}

En sendos trabajos, publicados con anterioridad en esta RevisTA, se analizaron tanto la distribución provincial y regional de la población española, como la evolución de la población de los Municipios españoles desde principios de siglo y su distribución geográfica según los resultados obtenidos en el Censo de 1970.

Uno de los trabajos capitales que realiza el Instituto Nacional de Estadística desde el año 1857 es la formación de los censos oficiales de población. Ultimamente ha publicado el tomo tercero del Censo de 1970, dedicado a las características de la población, en el que presenta los resultados nacionales. Esta publicación se complementa con las cifras provinciales que se contienen en 50 monografías, una por Provincia. 
En el prólogo-introducción a la publicación citada se exponen muy acertadamente los objetivos que se persiguen con la formación de los censos de población en todos los países, y que estimamos interesante sintetizar.

El primer objetivo consiste en el recuento de la población, en determinar el número de habitantes de cada Municipio y de cada entidad de población como base para fijar la población total del país y la que registran las Regiones históricas o naturales, las Provincias, las comarcas geográficas y las restantes áreas territoriales que sea necesario considerar en cada caso. Se fija de esta forma. la cifra legal de habitantes, que tiene cada día mayor número de efectos administrativos, en el orden estatal, local e institucional, en las relaciones de la Administración con los administrados.

El segundo objetivo es conocer la estructura y características de la población del país. La composición de la familia, las características personales de los habitantes, su nivel cultural y socio-económico, los movimientos migratorios y otros aspectos constituyen informaciones numéricas de extraordinaria importancia para el estudio de los problemas demográficos, sociales y económicos y para la planificación del desarrollo en los ámbitos nacional y local y para establecer las previsiones futuras del desarrollo o implantación de nuevos servicios públicos.

En tercer lugar, el censo de población constituye un marco adecuado para programar las encuestas por muestreo, que, en número siempre creciente, se han de realizar en un futuro inmediato, ya que sirven de base para calcular los parámetros de las distintas variables y facilitan la selección de las unidades estadísticas que han de componer la muestra. Igualmente ayudan a la formación de las estadísticas intercensales y a las investigaciones demográficas de proyecciones futuras.

Finalmente, cada país, al formar su censo de población, secunda un programa censal mundial, patrocinado por la Oficina de Estadística de las Naciones Unidas, tiene muy en cuenta las recomendaciones de la citada Oficina y de la Conferencia de Estadísticos Europeos, y sus resultados se facilitan a los organismos internacionales para garantizar la comparabilidad internacional de resultados estadísticos.

La riqueza informativa que contiene la mencionada monografía y las publicaciones provinciales hace imposible un análisis exhaustivo. Forzosamente hemos de limitarnos en este trabajo a un comen- 
tario de algunas características, las más trascendentes y significativas en el conjunto nacional, y salvo una excepción, tomaremos como base de la distribución geográfica las tres zonas con referencia a las cuales tradicionalmente presenta los resultados censales el Instituto Nacional de Estadística: la urbana, que comprende todos los poblados o entidades de población que tienen, en cada caso, más de 10.000 habitantes; la intermedia, que abarca las entidades de población comprendidas entre 2.001 y 10.000 habitantes, y la rural, en la que se contiene la población que reside en entidades de 2.000 habitantes como máximo.

\section{LA COMPOSICION DE LA FAMILIA ESPAÑOLA}

A la familia se la ha considerado como la "célula básica" de la sociedad, aun cuando parece advertirse una crisis de la institución en los momentos en que vivimos. A efectos censales se la ha definido como "el grupo de personas, vinculadas generalmente por parentesco, que hacen vida en común, ocupando normalmente la totalidad de una vivienda". Dentro de este amplio concepto de familia se incluye a los huéspedes no familiares a los que se cede el uso de una o más habitaciones o se les prestan ciertos servicios.

Toda familia tiene, a efectos censales, una "persona principal», y la relación que los demás miembros tienen con aquélla permite conocer la estructura de la familia, que aparece descrita en la hoja de inscripción censal, que contiene los datos primarios para la formación del censo de población.

Si tenemos en cuenta la población española de hecho en 1970, de 33.956.376 habitantes, y el número de familias registrado, que alcanza a 8.853.660, podemos fijar la composición media de la familia española en 3,8 personas.

Por zonas, la composición de la familia no difiere mucho, como se deduce de estas cifras:

\begin{tabular}{|c|c|c|c|}
\hline & $\begin{array}{l}\text { Número de } \\
\text { habitantes }\end{array}$ & $\begin{array}{l}\text { Numero de } \\
\text { familias }\end{array}$ & $\begin{array}{c}\text { Composición } \\
\text { media }\end{array}$ \\
\hline 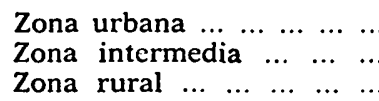 & $\begin{array}{r}18.819 .684 \\
6.615 .763 \\
8.520 .929\end{array}$ & $\begin{array}{l}4.826 .746 \\
1.747 .328 \\
2.279 .590\end{array}$ & $\begin{array}{l}3,9 \\
3,8 \\
3,7\end{array}$ \\
\hline
\end{tabular}


si bien se aprecia, en razón del concepto amplio utilizado, una matización de sólo una décima, que nos indica que en la zona urbana la familia aparece algo mayor que en la rural.

La distribución de los habitantes y de las familias es muy similar en las tres zonas. De cada 100 habitantes o familias, 54 se hallan en la zona urbana, 20 en la intermedia y 26 en la rural.

Atendiendo el número de personas que componen la familia, según zonas, se obtiene la siguiente distribución:

\begin{tabular}{|c|c|c|c|}
\hline & \multicolumn{3}{|c|}{ POR CENTA JES } \\
\hline & $\begin{array}{c}\text { Zona } \\
\text { urbana }\end{array}$ & $\begin{array}{c}\text { Zona } \\
\text { intermedia }\end{array}$ & $\begin{array}{l}\text { Zona } \\
\text { rural }\end{array}$ \\
\hline $\begin{array}{ccccccccccccc}\operatorname{De} u n a & \ldots & \ldots & \ldots & \ldots & \ldots & \ldots & \ldots & \ldots & \ldots & \ldots & \ldots & \ldots\end{array}$ & 6,5 & 7,7 & 9,3 \\
\hline $\operatorname{De} \operatorname{dos}\left[\begin{array}{llllllllllll}\ldots & \ldots & \ldots & \ldots & \ldots & \ldots & \ldots & \ldots & \ldots & \ldots & \ldots & \ldots\end{array}\right.$ & 17,9 & $17,9^{\circ}$ & 18,4 \\
\hline 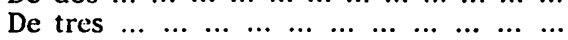 & 20,0 & 18,5 & 18,1 \\
\hline $\begin{array}{llllllllllll}\text { De cuatro } & \ldots & \ldots & \ldots & \ldots & \ldots & \ldots & \ldots & \ldots & \ldots & \ldots & \ldots\end{array}$ & 22,7 & 21,7 & 19,8 \\
\hline $\begin{array}{llllllllllll}\text { De } \operatorname{cinco} & \ldots & \ldots & \ldots & \ldots & \ldots & \ldots & \ldots & \ldots & \ldots & \ldots & \ldots\end{array}$ & 15,6 & 15,8 & 14,9 \\
\hline $\begin{array}{clllllllllll}\operatorname{De} \operatorname{seis} & \ldots & \ldots & \ldots & \ldots & \ldots & \ldots & \ldots & \ldots & \ldots & \ldots & \ldots\end{array}$ & 8,8 & 9,3 & 9,3 \\
\hline $\begin{array}{lllllllllll}\text { De siete } & \ldots & \ldots & \ldots & \ldots & \ldots & \ldots & \ldots & \ldots & \ldots & \ldots\end{array}$ & 4,4 & 4,7 & 5,0 \\
\hline $\begin{array}{llllllllllll}\text { De } \text { ocho } & \ldots & \ldots & \ldots & \ldots & \ldots & \ldots & \ldots & \ldots & \ldots & \ldots & \ldots\end{array}$ & 2,2 & 2,4 & 2,7 \\
\hline $\begin{array}{lllllllllll}\text { De nueve } & \ldots & \ldots & \ldots & \ldots & \ldots & \ldots & \ldots & \ldots & \ldots & \ldots\end{array}$ & 0,9 & 0,9 & 1,2 \\
\hline $\begin{array}{llllllllll}\text { De } \operatorname{diez~y~} \text { más } & \ldots & \ldots & \ldots & \ldots & \ldots & \ldots & \ldots & \ldots & \ldots\end{array}$ & 1,0 & 1,1 & 1,3 \\
\hline
\end{tabular}

y resumiéndola se obtiene ésta:

\begin{tabular}{|c|c|c|c|}
\hline & $\begin{array}{c}\text { Zona } \\
\text { urbana }\end{array}$ & $\begin{array}{c}\text { Zona } \\
\text { intermedia }\end{array}$ & $\begin{array}{l}\text { Zona } \\
\text { rural }\end{array}$ \\
\hline $\begin{array}{llllllllll}\operatorname{Hasta} \text { cuatro } & \ldots & \ldots & \ldots & \ldots & \ldots & \ldots & \ldots & \ldots & \ldots \\
\text { De cinco a ocho } & \ldots & \ldots & \ldots & \ldots & \ldots & \ldots & \ldots & \ldots \\
\text { Más de ocho } & \ldots & \ldots & \ldots & \ldots & \ldots & \ldots & \ldots & \ldots & \ldots\end{array}$ & $\begin{array}{r}67,1 \\
31,0 \\
1,9\end{array}$ & $\begin{array}{r}65,8 \\
32,2 \\
2,0\end{array}$ & $\begin{array}{r}65,6 \\
31,9 \\
2,5\end{array}$ \\
\hline
\end{tabular}

llegándose a la conclusión de que las dos terceras partes de las familias se componen de cuatro miembros como máximo; casi una tercera parte tienen de cinco a ocho miembros, y las más numerosas representan alrededor del 2 por 100. Las primeras predominan ligeramente en las zonas urbanas, y las restantes en las zonas intermedia y rural.

A las familias se las ha clasificado, en relación con el núcleo familiar, en esta forma: 


\begin{tabular}{|c|c|c|c|}
\hline \multirow[b]{3}{*}{ 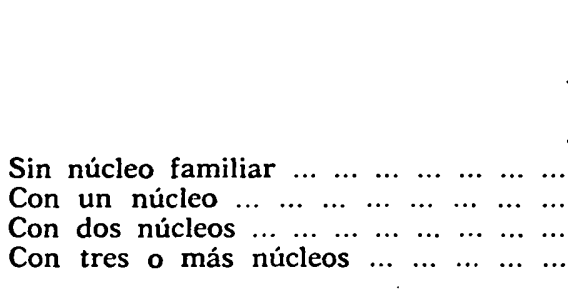 } & $\begin{array}{c}\text { Zona } \\
\text { urbana }\end{array}$ & $\begin{array}{c}\text { Zona } \\
\text { intermedia }\end{array}$ & $\begin{array}{l}\text { Zona } \\
\text { rural }\end{array}$ \\
\hline & \multicolumn{3}{|c|}{ CIFRAS ABSOLUTAS } \\
\hline & $\begin{array}{r}490.519 \\
4.098 .566 \\
231.145 \\
6.516\end{array}$ & $\begin{array}{r}174.794 \\
1.476 .925 \\
92.554 \\
3.055\end{array}$ & $\begin{array}{r}274.741 \\
1.822 .213 \\
173.111 \\
9.525\end{array}$ \\
\hline & \multicolumn{3}{|c|}{ POR CENTAJES } \\
\hline $\begin{array}{lllllllll}\text { Sin núcleo familiar } & \ldots & \ldots & \ldots & \ldots & \ldots & \ldots & \ldots \\
\text { Con un } \text { núcleo } & \ldots & \ldots & \ldots & \ldots & \ldots & \ldots & \ldots & \ldots \\
\text { Con dos núcleos } & \ldots & \ldots & \ldots & \ldots & \ldots & \ldots & \ldots \\
\text { Con tres } 0 \text { más } & \ldots & \ldots & \ldots & \ldots & \ldots & \ldots & \ldots & \ldots\end{array}$ & $\begin{array}{r}10,2 \\
84,9 \\
4,8 \\
0,1\end{array}$ & $\begin{array}{r}10,0 \\
84,5 \\
5,3 \\
0,2\end{array}$ & $\begin{array}{r}12,1 \\
79,9 \\
7,6 \\
0,4\end{array}$ \\
\hline
\end{tabular}

El caso normal, como se puede apreciar, es el de la familia con un núcleo familiar, que corresponde a cuatro de cada cinco familias y que está constituido por un matrimonio con o sin hijos solteros o un padre o una madre con hijos solteros. En algo más de la sexta parte de los casos conviven con otras personas que no pertenecen al núcleo y que no pertenecen al servicio doméstico. Las familias con servicio doméstico suponen solamente el 1,5 por 100 , porcentaje que se reduce en las zonas intermedia y rural.

Las familias con dos o más núcleos emparentados, en general convivencia de padres e hijos, suponen del 5 al 8 por 100 del total y tienen mayor entidad en las zonas intermedia y rural. Las familias sin núcleo familiar alcanzan el 10 por 100 del total; en la mayor parte de los casos se trata de personas que viven solas, pero se registra en casi la cuarta parte de ellas la convivencia con otras personas emparentadas o no, pero en ningún caso con núcleos familiares.

La distribución por zonas de los cuatro tipos de núcleos considerados ofrece este detalle:

\begin{tabular}{|c|c|c|c|}
\hline & $\begin{array}{c}\text { Zona } \\
\text { urbana }\end{array}$ & $\begin{array}{c}\text { Zona } \\
\text { intermedia }\end{array}$ & $\begin{array}{l}\text { Zona } \\
\text { rural }\end{array}$ \\
\hline & \multicolumn{3}{|c|}{ CIFRAS ABSOLUTAS } \\
\hline 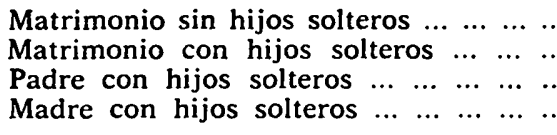 & $\begin{array}{r}903.364 \\
3.231 .360 \\
84.152 \\
361.524\end{array}$ & $\begin{array}{r}327.992 \\
1.191 .070 \\
36.246 \\
115.885\end{array}$ & $\begin{array}{r}469.496 \\
1.494 .168 \\
59.037 \\
174.317\end{array}$ \\
\hline & \multicolumn{3}{|c|}{ PORCENTAJES } \\
\hline 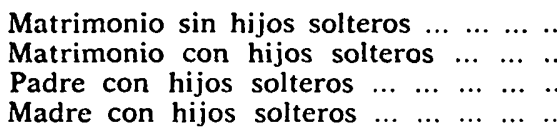 & $\begin{array}{r}19,7 \\
70,6 \\
1,8 \\
7,9\end{array}$ & $\begin{array}{r}19,6 \\
71,3 \\
2,2 \\
6,9\end{array}$ & $\begin{array}{r}21,4 \\
68,0 \\
2,7 \\
.7,9\end{array}$ \\
\hline
\end{tabular}


El caso más frecuente es el del matrimonio con hijos solteros, que corresponde a 70 de cada 100 núcleos familiares. El matrimonio sin hijos solteros representa el 20 por 100; el núcleo formado por el padre con hijos solteros es poco frecuente, pero lo es más el de la madre con hijos solteros, debido al mayor número de viudas, más de cuatro veces superior al de viudos. Es de resaltar el mayor peso de los matrimonios sin hijos en la zona rural, y lo propio ocurre con los padres sin hijos.

No viven en familia 620.010 personas, que equivalen al 1,8 por 100 de la población total. Se trata de quienes en el momento censal vivían en hoteles y residencias no hoteleras, internados docentes, sanatorios o centros benéficos, en centros penitenciarios, formaban parte de comunidades religiosas o se hallaban en cuarteles, barcos de guerra o campamentos. El 58,1 por 100 de ellos eran varones. Este colectivo tiene mayor peso en las capitales de Provincia y en la zona urbana, llegando al 3 por 100 en las primeras y superando el 2 por 100 en la última, mientras que en las zonas intermedia y rural alcanza el 1,1 y el 1,5 por 100, respectivamente.

\section{LAS CARACTERISTICAS PERSONALES: EL SEXO, EL ESTADO CIVIL, LA EDAD Y LA NACIONALIDAD}

En razón del sexo, la población española se descompone en 16.575.164 varones y 17.381.212 mujeres, que suponen respectivamente el 48,8 y el 51,2 por 100 . Hay un ligero predominio del sexo femenino, que se pone igualmente de manifiesto en todas las zonas:

\begin{tabular}{|c|c|c|}
\hline \multirow[b]{3}{*}{$\begin{array}{llllllll}\text { Zona urbana } & \ldots & \ldots & \ldots & \ldots & \ldots & \ldots & \ldots \\
\text { Zona intermedia } & \ldots & \ldots & \ldots & \ldots & \ldots \\
\text { Zona } \operatorname{rural} & \ldots & \ldots & \ldots & \ldots & \ldots & \ldots & \ldots\end{array}$} & Varones & Mujeres \\
\hline & \multicolumn{2}{|c|}{ CIFRAS ABSOLUTAS } \\
\hline & $\begin{array}{l}9.064 .918 \\
3.259 .968 \\
4.250 .278\end{array}$ & $\begin{array}{l}9.754 .766 \\
3.355 .795 \\
4.270 .651\end{array}$ \\
\hline & \multicolumn{2}{|c|}{ PORCENTAJES } \\
\hline 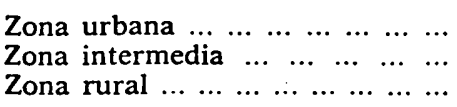 & $\begin{array}{l}48,2 \\
49,3 \\
49,9\end{array}$ & $\begin{array}{l}51,8 \\
50,7 \\
50,1\end{array}$ \\
\hline
\end{tabular}


tendiendo hacia un mayor equilibrio en las zonas intermedia y rural. Por el contrario, en las capitales de Provincia se acentúa la desproporción en favor de las mujeres, que llegan al 52,3 por 100.

En el conjunto nacional, hasta los veinticuatro años predominan los varones, y en las edades siguientes van manifestándose en mayor número las mujeres, acentuándose la desproporción numéricamente a medida que se avanza en la tabla de edades.

En cuanto al estado civil, combinado con el sexo, hemos elaborado esta distribución:

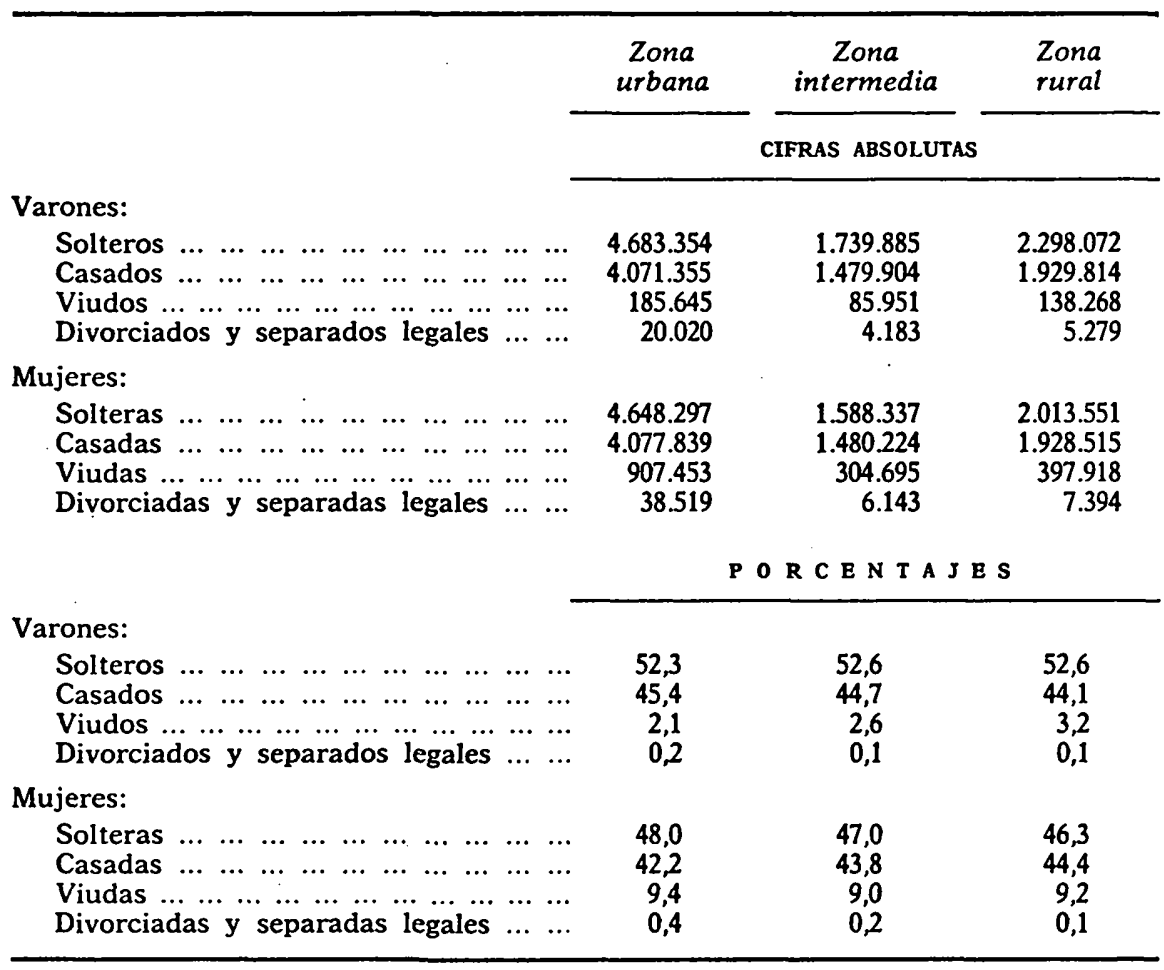

En tanto que de cada 100 varones en la zona urbana son solteros 52, casados 45 y viudos dos, respecto al sexo femenino, de cada 100 mujeres, sólo son solteras 48,42 casadas y, sin embargo, las viudas alcanzan el 9 por 100. Es de subrayar este mayor número de viudas sobre viudos en proporción de cuatro a uno, y la mayor proporción de viudos en la zona rural. Igualmente es de resaltar el mayor peso de los casados en la zona urbana y de las casadas en las otras dos zonas, y la cifra de viudos en las zonas intermedia 
y rural. Los divorciados y separados legales, con bajos porcentajes, destacan en la zona urbana, con referencia a ambos sexos.

La distribución por edades de la población de un país es altamente significativa. Expresa la influencia de las tendencias de natalidad y de la mortalidad y permite determinar el volumen futuro de la población activa.

En los censos que se citan, ofrece este detalle:

\begin{tabular}{|c|c|c|c|}
\hline & \multicolumn{3}{|c|}{ TANTO POR 1.000} \\
\hline & 1930 & 1950 & 1970 \\
\hline 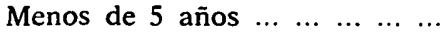 & 110,1 & 91,6 & 94,7 \\
\hline 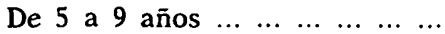 & 109,2 & 87,0 & 94,8 \\
\hline De 10 a 14 años $\ldots \ldots$ & 104,0 & 83,2 & 89,2 \\
\hline 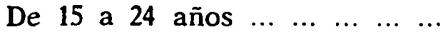 & 183,7 & 191,6 & 153,7 \\
\hline De 25 a 34 años $\ldots \ldots \ldots \ldots \ldots$ & 151,0 & 154,5 & 125,5 \\
\hline De 35 a 44 años $\ldots \ldots \ldots$ & 117,6 & 131,8 & 138,1 \\
\hline 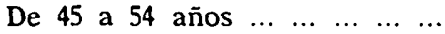 & 95,9 & 109,4 & 113,7 \\
\hline 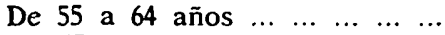 & 73,4 & 77,9 & 92,7 \\
\hline 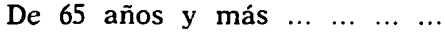 & 55,1 & 72,8 & 97,6 \\
\hline
\end{tabular}

La tendencia al descenso de la natalidad que se presenta en España, al igual que en la mayor parte de los países europeos se trunca antes de 1960 y se inicia un moderado incremento, que se refleja en las cifras anteriores, al comparar los tres primeros grupos de la tabla. Se aprecia, por el contrario, la disminución en los grupos de quince a treinta y cuatro años, reflejo, en parte, de la baja natalidad inmediata anterior y se manifiesta cómo aumenta el número de españoles de edades más altas de la edad adulta y de la vejez.

Con referencia al año 1970, la distribución en los tres grupos clásicos, para el conjunto nacional y para las tres zonas consideradas, es como sigue:

\begin{tabular}{|c|c|c|c|c|}
\hline & \multicolumn{4}{|c|}{ TANTO POR 1.000} \\
\hline & $\begin{array}{l}\text { Conjunto } \\
\text { nacional }\end{array}$ & $\begin{array}{l}\text { Zona } \\
\text { urbana }\end{array}$ & $\begin{array}{c}\text { Zona } \\
\text { intermedia }\end{array}$ & $\begin{array}{l}\text { Zona } \\
\text { rural }\end{array}$ \\
\hline Hasta 24 años ... & 432,4 & 439,2 & 440,5 & 411,4 \\
\hline De 25 a 64 años ... & 470,0 & 475,8 & 459,5 & 469,5 \\
\hline 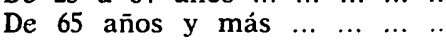 & 97,6 & 85,0 & 100,0 & 119,1 \\
\hline
\end{tabular}

Las tasas más altas en el grupo primero, de edades jóvenes, corresponden a las zonas intermedia y urbana. La población activa, 
que básicamente corresponde al segundo grupo, destaca en la zona urbana, con la máxima, y en la rural, y se aprecia como un vacío relativo en la zona intermedia. En el tercer grupo, el de viejos, se aprecia claramente un predominio de ellos en la zona rural, sobre todo, y en la intermedia.

La zona urbana parece que se presenta en mejores condiciones al contar con la más alta tasa de población activa y con gentes de edades jóvenes por encima del promedio nacional, y por el contrario, el menor peso de la población vieja, en edades de retiro y no actividad. Por el contrario, es la zona rural la menos favorecida, pues ofrece un déficit de jóvenes, una carga, la mayor, de viejos y una población activa inferior a la nacional.

\section{EL LUGAR DE PROCEDENCIA DE LA POBLACION RESIDENTE}

La distribución en el conjunto nacional de la población censada, según el lugar de su nacimiento, es ésta:

\begin{tabular}{|c|c|}
\hline & Porcentajes \\
\hline $\begin{array}{llllllll}\text { Nacidos en España } & \ldots & \ldots & \ldots & \ldots & \ldots & \ldots & \ldots\end{array}$ & 98,7 \\
\hline En la misma Provincia en que se censaron ... & 75,5 \\
\hline En los mismos Municipios en que se censaron & 58,5 \\
\hline En otros Municipios de la misma Provincia & 17,0 \\
\hline 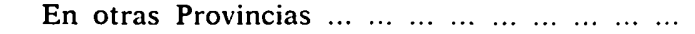 & 23,2 \\
\hline 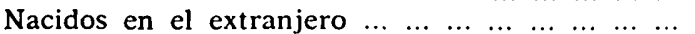 & 1,3 \\
\hline
\end{tabular}

La síntesis anterior refleja una situación real. Se puede afirmar que de cada 100 habitantes censados en 1970,58 no se habían movido del Municipio de su nacimiento, y habian pasado a residir a otros Municipios 42. Conviene distinguir, sin embargo, entre el movimiento intraprovincial, que no rebasa los límites de la Provincia donde se nació, y el movimiento interprovincial, que supone el cambio de residencia a otras Provincias. De los 42 habitantes de cada 100 que no residian en el Municipio de nacimiento, 17 habían pasado a residir a otros Municipios de la misma Provincia, algo más de la sexta parte de la población española. Mayor entidad ha tenido la emigración a otras Provincias, pues viene a representar la cuarta parte de la población total.

En resumen, puede afirmarse que uno de cada cuatro habitantes ha emigrado a Provincia distinta de la de su nacimiento y que uno 
de cada seis pasó a residir a otro Municipio distinto del de su nacimiento dentro de la misma Provincia.

Las cifras comentadas dan de por sí idea del volumen cuantitativo de los movimientos migratorios habidos en los últimos decenios, que viene a resumirse, en sus efectos, en la síntesis analizada, con referencia a 1970. Es, en verdad, el resultado de unos cambios de población que se han producido durante más de una generación.

Entre las tablas elaboradas en el censo de 1970 aparece una que estimamos muy interesante, en la que se presenta clasificada a la población de diez y más años según el lugar de residencia en 1960 y en 1970. El hecho de que se refiera sólo al último decenio y no a toda la población, limita cuantitativamente sus efectos. La referida información afecta a 27.514 .980 habitantes, de los cuales:

\begin{tabular}{|c|c|c|}
\hline & $\begin{array}{l}\text { Numero de } \\
\text { habitantes }\end{array}$ & Porcentajes \\
\hline 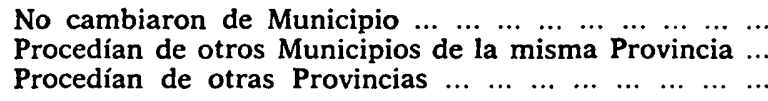 & $\begin{array}{r}23.050 .619 \\
1.703 .040 \\
2.761 .321\end{array}$ & $\begin{array}{r}83,8 \\
6,2 \\
10,0\end{array}$ \\
\hline
\end{tabular}

Atendiendo al lugar de nacimiento de la población censada en 1970 se llegaba a la conclusión de que 5,8 millones de habitantes residian en Municipio distinto al de su nacimiento, pero dentro de la misma Provincia; que 7,8 millones de españoles residian en otras Provincias distintas a las de su nacimiento, y que 400.000 habian nacido en el extranjero, dando un total de 14 millones no residentes en el mismo Municipio de su nacimiento.

En la tabla a que acabamos de aludir sólo se atiende a la comparación entre el Municipio de residencia en 1970 y en 1960 para las personas censadas en el primero de los años citados, y de otra parte, la información se limita a los mayores de diez años. De aquí que las cifras de desplazados dentro de la misma Provincia y de los procedentes de otras sean muy inferiores. Comprenden, en total, 4,5 millones de españoles, de los que casi tres millones constituyeron la masa migratoria interprovincial, y 1,7 millones promovieron cambios de residencia dentro de cada Provincia.

En la tabla que se transcribe a continuación se presentan, detalladas por Provincias, las cifras correspondientes, tanto absolutas como los porcentajes expresivos de los valores de los citados desplazamientos para cada una de ellas. 
ESTADISTICA

737

POBLACION DE DIEZ Y MAS AÑOS EN 1970

\begin{tabular}{|c|c|c|c|c|c|c|c|}
\hline PROVINCIAS & $\begin{array}{c}\text { Total } \\
\text { (a) }\end{array}$ & $\begin{array}{l}\text { No cambiaron } \\
\text { de Municipio } \\
\text { desde } 1960 \\
\text { (b) }\end{array}$ & $\begin{array}{l}\text { Procedian de } \\
\text { otros } \\
\text { Municipios de } \\
\text { la misma } \\
\text { Provincia (c) }\end{array}$ & $\begin{array}{c}\text { Que cambiaron } \\
\text { de residencia } \\
\text { desde } 1960 \text { y } \\
\text { procedian de } \\
\text { otras Provin- } \\
\text { cias (d) }\end{array}$ & $\frac{(b)}{(a)} 100$ & $\frac{(c)}{(a)} 100$ & $\frac{(d)}{(a)} 100$ \\
\hline 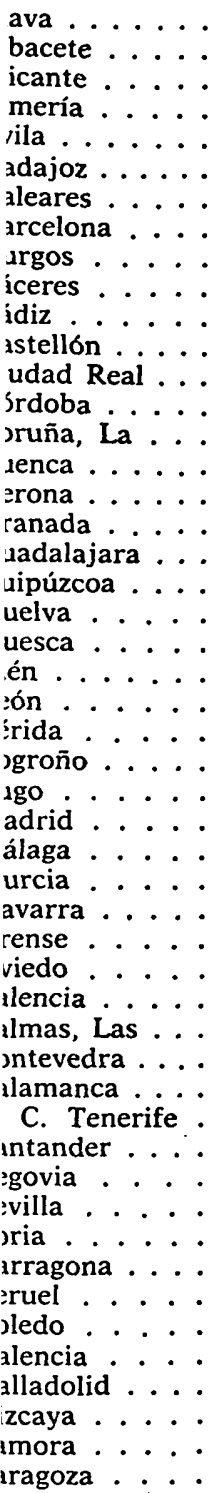 & $\begin{array}{r}158.721 \\
271.422 \\
739.817 \\
299.297 \\
178.691 \\
567.043 \\
440.390 \\
3.202 .990 \\
300.317 \\
382.764 \\
671.612 \\
322.928 \\
413.438 \\
579.143 \\
861.300 \\
208.253 \\
345.459 \\
583.814 \\
126.037 \\
501.869 \\
324.488 \\
189.070 \\
525.886 \\
468.177 \\
291.582 \\
196.824 \\
367.200 \\
3.018 .499 \\
667.608 \\
656.146 \\
382.155 \\
380.971 \\
881.696 \\
168.597 \\
409.081 \\
636.413 \\
315.356 \\
448.361 \\
385.765 \\
133.146 \\
1.045 .171 \\
99.570 \\
361.919 \\
149.343 \\
392.678 \\
1.443 .361 \\
333.131 \\
835.772 \\
219.768 \\
631.942\end{array}$ & $\begin{array}{r}115.633 \\
247.751 \\
603.196 \\
264.821 \\
167.336 \\
525.528 \\
373.811 \\
2.301 .494 \\
253.025 \\
347.950 \\
616.985 \\
270.512 \\
387.582 \\
532.362 \\
784.021 \\
192.641 \\
274.877 \\
526.970 \\
107.780 \\
387.595 \\
297.054 \\
160.494 \\
492.178 \\
404.730 \\
249.591 \\
166.115 \\
349.777 \\
2.229 .823 \\
587.102 \\
607.382 \\
306.383 \\
360.412 \\
743.472 \\
144.786 \\
351.865 \\
586.344 \\
274.274 \\
393.274 \\
331.679 \\
117.573 \\
925.390 \\
87.344 \\
303.654 \\
136.099 \\
362.247 \\
1.191 .184 \\
262.792 \\
631.195 \\
202.192 \\
512.344\end{array}$ & $\begin{array}{r}8.572 \\
14.916 \\
37.041 \\
18.737 \\
5.761 \\
27.749 \\
20.126 \\
270.059 \\
26.786 \\
24.700 \\
24.224 \\
18.827 \\
15.057 \\
27.953 \\
46.311 \\
10.251 \\
33.443 \\
35.827 \\
10.432 \\
37.015 \\
16.060 \\
17.700 \\
21.166 \\
44.035 \\
19.789 \\
16.585 \\
10.330 \\
129.806 \\
31.937 \\
23.268 \\
38.231 \\
9.188 \\
87.590 \\
13.726 \\
35.266 \\
21.439 \\
25.081 \\
35.481 \\
31.440 \\
9.603 \\
61.370 \\
8.400 \\
14.241 \\
7.026 \\
16.600 \\
88.705 \\
29.611 \\
70.476 \\
11.074 \\
46.029\end{array}$ & $\begin{array}{r}34.516 \\
8.755 \\
99.580 \\
15.739 \\
5.594 \\
13.766 \\
46.453 \\
631.437 \\
20.506 \\
10.114 \\
30.403 \\
33.589 \\
10.799 \\
18.828 \\
30.968 \\
5.361 \\
39.139 \\
21.017 \\
7.825 \\
77.259 \\
11.374 \\
10.876 \\
12.542 \\
19.412 \\
22.202 \\
14.124 \\
7.093 \\
658.870 \\
48.569 \\
25.496 \\
37.541 \\
11.371 \\
50.634 \\
10.085 \\
21.950 \\
28.630 \\
16.001 \\
19.606 \\
22.646 \\
5.970 \\
58.411 \\
3.826 \\
44.024 \\
6.217 \\
13.831 \\
163.472 \\
40.728 \\
134.101 \\
6.502 \\
73.569\end{array}$ & $\begin{array}{l}72,9 \\
91,3 \\
81,5 \\
88,5 \\
93,6 \\
92,7 \\
84,9 \\
71,9 \\
84,3 \\
90,9 \\
91,9 \\
83,8 \\
93,7 \\
91,9 \\
90,1 \\
92,5 \\
79,6 \\
90,3 \\
85,5 \\
77,2 \\
91,5 \\
84,9 \\
93,6 \\
86,4 \\
85,6 \\
84,4 \\
95,3 \\
73,9 \\
88,0 \\
92,6 \\
80,2 \\
94,8 \\
84,3 \\
85,9 \\
86,0 \\
92,1 \\
87,0 \\
87,7 \\
86,0 \\
88,3 \\
88,6 \\
87,9 \\
83,9 \\
91,2 \\
92,3 \\
82,5 \\
78,3 \\
75,5 \\
92,0 \\
81,1\end{array}$ & $\begin{array}{r}5,4 \\
5,5 \\
5,0 \\
6,3 \\
3,2 \\
4,9 \\
4,6 \\
8,4 \\
8,9 \\
6,5 \\
3,6 \\
5,8 \\
3,6 \\
4,8 \\
5,3 \\
4,9 \\
9,1 \\
6,1 \\
8,3 \\
7,4 \\
5,0 \\
9,4 \\
4,0 \\
9,4 \\
6,8 \\
8,4 \\
2,8 \\
4,3 \\
4,8 \\
3,5 \\
10,0 \\
2,4 \\
10,0 \\
8,1 \\
8,6 \\
3,4 \\
8,0 \\
7,9 \\
8,1 \\
7,3 \\
5,9 \\
8,4 \\
3,9 \\
4,6 \\
4,2 \\
6,2 \\
8,9 \\
8,5 \\
5,0 \\
7,3\end{array}$ & $\begin{array}{r}21,7 \\
3,2 \\
13,5 \\
5,2 \\
3,2 \\
2,4 \\
10,5 \\
19,7 \\
6,8 \\
2,6 \\
4,5 \\
10,4 \\
2,7 \\
3,3 \\
3,6 \\
2,6 \\
11,3 \\
3,6 \\
6,2 \\
15,4 \\
3,5 \\
5,7 \\
2,4 \\
4,2 \\
7,6 \\
7,2 \\
1,9 \\
21,8 \\
7,2 \\
3,9 \\
9,8 \\
2,8 \\
5,7 \\
6,0 \\
5,4 \\
4,5 \\
5,0 \\
4,4 \\
5,9 \\
4,4 \\
5,5 \\
3,7 \\
12,2 \\
4,2 \\
3,5 \\
11,3 \\
12,2 \\
16,0 \\
3,0 \\
11,6\end{array}$ \\
\hline
\end{tabular}


En primer término conviene examinar los desplazamientos entre distintas Provincias, que afectan básicamente a 13 Provincias, como receptoras de las masas migrantes, con porcentajes superiores al promedio nacional. En cifras absolutas ocupan los dos primeros lugares Madrid y Barcelona, que han recibido en los diez años intercensales más de 600.000 habitantes cada una; a nivel bastante inferior se sitúan Valencia, Vizcaya y Alicante. En términos relativos los dos primeros lugares corresponden a Madrid y Alava, seguidas de Barcelona, Vizcaya y Guipúzcoa, como las de mayor importancia.

Alava recibe inmigrantes procedentes de Vizcaya, Guipúzcoa, Navarra y Logroño, Burgos, Palencia, Salamanca, Zamora y de Cáceres principalmente.

La zona de atracción de Guipúzcoa es más amplia; aparte de las otras Provincias vascongadas, Navarra y Logroño, Provincias del valle del Duero, como Burgos, Valladolid, León, Palencia, Salamanca, Zamora, Avila y Segovia, le suministran inmigrantes las dos Provincias extremeñas, La Coruña, Orense y Pontevedra, Màdrid y Córdoba.

Aún es más amplia la zona a que se extiende la influencia de Vizcaya, pues aparte de las citadas con respecto a Guipúzcoa, recibe inmigrantes de Lugo, Oviedo, Santander, Soria, Zaragoza y las restantes Provincias andaluzas.

Gerona recibe inmigrantes de Barcelona, Extremadura y Andalucía.

Barcelona es uno de los grandes focos de atracción; todas las Provincias españolas le son tributarias, pero especialmente las otras Provincias catalanas, Aragón, Madrid, Ciudad Real, Cuenca y. Extremadura, además de Murcia y Lugo.

Tarragona recibe población de Barcelona y Lérida, Castellón, Valencia, Teruel y Zaragoza, Madrid, Ciudad Real, Badajoz y algunas Provincias andaluzas.

Castellón, con un ámbito más restringido que se limita a Barcelona y Tarragona, Teruel y Valencia, Cuenca y Albacete, Ciudad Real, Granada y Jaén.

A Valencia le nutren de habitantes especialmente Albacete, Cuenca, Ciudad Real y Jaén, y secundariamente de sus limítrofes: Castellón, Alicante, Baleares, Murcia, Extremadura y Andalucía, Toledo y Zaragoza.

Alicante recibe inmigrantes de las limítrofes: Albacete, Murcia, Ciudad Real y Cuenca, Valencia, Extremadura y Andalucía. 
A Baleares llegan gentes procedentes de Barcelona, Valencia, Murcia y Albacete, Extremadura y Andalucía.

Madrid atrae población principalmente de sus Provincias limítrofes: Toledo, Ciudad Real, Cuenca, Guadalajara, Avila, Segovia, Extremadura, Córdoba, otras varias del valle del Duero y Oviedo, si bien todas las Provincias contribuyen, aunque en forma desigual, al incremento de su población, ya que esta Provincia constituye el primer foco de atracción demográfica del país.

Valladolid tiene una zona de influencia demográfica reducida a las Provincias del valle del Duero, Vizcaya y Oviedo.

Y Zaragoza recibe inmigrantes de Teruel y Huesca, Barcelona y Lérida, Logroño y Navarra, Burgos, Guadalajara y Soria, Valencia, Madrid y Córdoba especialmente.

Se aprecia cómo las Provincias vascongadas reciben población inmigrante de las Provincias limítrofes, del valle del Duero y de Galicia y Extremadura. Las Provincias del litoral mediterráneo también reciben, en primer término, habitantes de las limítrofes, de Castilla la Nueva y Extremadura y Andalucía. Madrid y Barcelona ejercen fuerte presión demográfica sobre toda España.

En definitiva, las 13 Provincias enumeradas, que atraen los mayores contingentes de inmigrantes en el interior del país, son, en líneas generales, las que contienen las principales zonas industriales y las aglomeraciones urbanas más importantes.

En cuanto a los desplazamientos intraprovinciales, ocupan los primeros lugares Oviedo y Navarra, juntamente con 22 Provincias más, que se extienden por la Meseta, el valle del Ebro, Cataluña, las Vascongadas, la zona cantábrica, Canarias y las Provincias de Valencia y Almería. Entre ellas figuran Provincias de economía preferentemente agraria, industrial y de servicios, y estos desplazamientos responden a la despoblación de los pequeños núcleos y a las corrientes que se dirigen hacia núcleos más importantes, y responden a causas más bien comarcales que nacionales, si bien buscan los mercados de trabajo en pequeñas áreas.

\section{EL ANALFABETISMO Y EL NIVEL DE INSTRUCCION DE LOS HABITANTES}

Se trata de dos características de suma importancia, por cuanto reflejan el nivel cultural del país. Sobre ellas se presenta en la monografía que comentamos en esta crónica una información nu- 
mérica muy significativa centrada en la tasa de analfabetismo, en el nivel de estudios alcanzado por la población censada en 1970 que no cursaba estudios y en el nivel de instrucción de los que cursaban estudio en el momento censal.

\section{El ANALFABETISMO}

La tasa de analfabetos para la población de diez y más años, según las normas de la UNESCO, ha sido en 1970 del 8,8 por 100, presentando los varones una tasa más favorable del 5,1, y las mujeres una tasa más elevada del 12,3.

En las capitales de Provincia se ofrece la situación más favorable, ya que la tasa general de sus analfabetos queda en el 5,4, la de los varones en el 2,6 y la de mujeres en el 7,9.

Las tasas que representan, para cada sexo, las tres zonas que venimos considerando son:

\begin{tabular}{|c|c|c|}
\hline & Varones & Mujeres \\
\hline $\begin{array}{llllllllll}\operatorname{Zn} & \\
\text { Zona } \operatorname{urbana} & \ldots & \ldots & \ldots & \ldots & \ldots & \ldots & \ldots & \ldots & \ldots \\
\text { Zona intermedia } & \ldots & \ldots & \ldots & \ldots & \ldots & \ldots & \ldots \\
\text { Zona } \operatorname{rural} & \ldots & \ldots & \ldots & \ldots & \ldots & \ldots & \ldots & \ldots & \ldots\end{array}$ & $\begin{array}{l}3,5 \\
7,7 \\
6,3\end{array}$ & $\begin{array}{r}9,7 \\
16,7 \\
14,5\end{array}$ \\
\hline
\end{tabular}

Es evidente que las altas tasas de analfabetismo del pasado han desaparecido. La situación ha mejorado de manera muy acusada. Las tasas anotadas son expresión de una situación favorable, pero en ellas tiene todavía un peso notable el analfabetismo de los grupos de edad más elevados. Ello se deduce fácilmente de los siguientes porcentajes:

\begin{tabular}{|c|c|c|c|c|c|c|}
\hline \multirow{2}{*}{ EDADES } & \multicolumn{2}{|c|}{ ZONA URBANA } & \multicolumn{2}{|c|}{ ZONA INTERMEDIA } & \multicolumn{2}{|c|}{ ZONA RURAL } \\
\hline & Varones & Mujeres & Varones & Mujeres & Varones & Mujeres \\
\hline De 10 a 14 años $\ldots \ldots \ldots \ldots$ & 0,5 & 0,5 & 0,7 & 0,1 & 0,6 & 0,4 \\
\hline De 15 a 19 años $\ldots \ldots \ldots \ldots$ & 1,4 & 1,7 & 2,6 & 2,5 & 1,8 & 1,6 \\
\hline De 20 a 24 años $\ldots \ldots \ldots \ldots$ & 1,3 & 2,3 & 2,4 & 3,8 & 1,8 & 2,5 \\
\hline De 25 a 29 años $\ldots \ldots \ldots \ldots$ & 1,5 & 3,6 & 2,9 & 6,7 & 2,3 & 4,6 \\
\hline De 30 a 34 años $\ldots \ldots \ldots \ldots$ & 2,4 & 6,6 & 5,0 & 11,8 & 3,8 & 8,3 \\
\hline De 35 a 44 años $\ldots \ldots \ldots \ldots$ & 3,3 & 8,4 & 6,8 & 14,8 & 4,8 & 10,2 \\
\hline De 45 a 54 años $\ldots \ldots \ldots \ldots$ & 3,9 & 10,3 & 8,4 & 17,8 & 6,2 & 13,5 \\
\hline De 55 a 64 años $\ldots \ldots \ldots \ldots$ & 6,0 & 18,0 & 12,7 & 29,7 & 10,2 & 24,4 \\
\hline De 65 años y más ... ... & 12,7 & 17,7 & 25,5 & 19,1 & 19,8 & 40,9 \\
\hline
\end{tabular}


Las tasas generacionales de analfabetismo son bien distintas. Se aprecia cómo en los grupos de edades jóvenes estas tasas son más bajas para cada zona que las generales, antes consignadas. Los españoles censados en 1970, de diez a cuarenta y cuatro años, ofrecen unas tasas muy débiles de analfabetismo, inferior al 3,5 por $100,7,7$ ó 6,3 , propios de las zonas consideradas respectivamente. Fenómeno semejante se aprecia en las mujeres y para los mismos grupos de edades, aunque en la zona rural se da la circunstancia favorable de que el grupo generacional de tasas mínimas o más bajas se amplía hasta los cincuenta y cuatro años.

La discriminación por sexos está bien marcada. En general el analfabetismo se manifiesta más claramente en la mujer, pero hay excepciones en algún grupo de edad. El mero transcurso del tiempo es evidente que ha de determinar una baja muy sensible en las tasas de analfabetismo de los grupos más altos de edades, que naturalmente ha de repercutir en una disminución general de la tasa de analfabetismo, tendente claramente a la desaparición de este fenómeno negativo en nuestro país.

2. Niveles dE estudio alcanzados por la población QUE No CURSABA ESTUDIOS EN EL MOMENTO CENSAL

Nos limitamos a consignar los datos en porcentajes en esta forma:

NIVELES DE ENSENAANZA

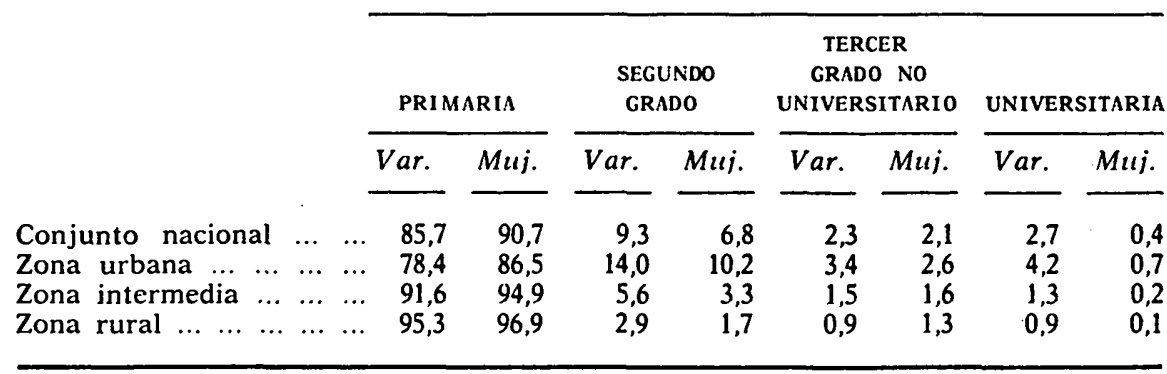

En el conjunto nacional, de cada 100 varones, poseen estudios primarios 86; estudios medios, nueve; de tercer grado no universitario, equivalente a nuestros antiguos peritajes, dos, y estudios 
universitarios, tres. En cuanto a las mujeres, la distribución difiere bastante en sentido desfavorable, dado que de cada 100 tienen estudios primarios 91; estudios medios, siete; el mismo nivel que los varones en estudios de tercer grado no universitarios, aunque de distinta naturaleza al de aquéllos, pero en estudios universitarios no llegan al 1 por 100 .

En la zona urbana la situación es superior a la del conjunto nacional para cada sexo y nivel de estudios. La situación aparece deteriorada en la zona intermedia y presenta las características más dramáticas en la zona rural, si bien conviene destacar que durante mucho tiempo en las zonas no urbanas del país los españoles no tenían las mismas posibilidades de estudio que en las grandes aglomeraciones urbanas. Sin embargo, en los últimos decenios ello ha ido haciéndose posible; $y$ así, en el cuadro de distribución por edades que consignamos en el estudio del analfabetismo se daba la circunstancia de que la zona rural aparece con tasas más favorables incluso que la intermedia para algunos grupos de edades. La cultura se ha extendido últimamente a todos los lugares y a todos los estratos del país.

Será muy aleccionador comparar esta última distribución con la que nos da la de

3. Niveles de estudio de los estudiantes en el momento CENSAl

La situación es la siguiente:

\begin{tabular}{|c|c|c|c|c|c|c|c|c|}
\hline & \multicolumn{2}{|c|}{ PRIMARIA } & \multicolumn{2}{|c|}{$\begin{array}{l}\text { SEGUNDO } \\
\text { GRADO }\end{array}$} & \multicolumn{2}{|c|}{$\begin{array}{c}\text { TERCER } \\
\text { GRADO NO } \\
\text { UNIVERSITARIO }\end{array}$} & \multicolumn{2}{|c|}{ UNIVERSITARIA } \\
\hline - & Var. & Muj. & Var. & Muj. & Var. & Muj. & Var. & Muj. \\
\hline 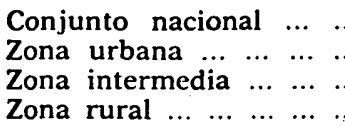 & $\begin{array}{ll}\ldots & 66,4 \\
\ldots & 60,2 \\
\ldots & 73,9 \\
\ldots & 76,8\end{array}$ & $\begin{array}{l}72,3 \\
66,5 \\
79,3 \\
81,6\end{array}$ & $\begin{array}{l}27,6 \\
32,0 \\
22,4 \\
19,8\end{array}$ & $\begin{array}{l}24,8 \\
29,8 \\
18,8 \\
16,8\end{array}$ & $\begin{array}{l}2,2 \\
2,6 \\
1,7 \\
1,6\end{array}$ & $\begin{array}{l}1,3 \\
1,4 \\
1,1 \\
1,1\end{array}$ & $\begin{array}{l}3,8 \\
5,2 \\
2,0 \\
1,8\end{array}$ & $\begin{array}{l}1,6 \\
2,3 \\
0,8 \\
0,5\end{array}$ \\
\hline
\end{tabular}

Conviene advertir que tanto en este cuadro como en el anterior no se recogen un pequeño grupo de habitantes cuyos estudios no han podido clasificarse. 
Se advierte, al comparar este cuadro con el anterior, una baja acusada en el porcentaje de estudiantes que cursan estudios primarios, y ello obedece especialmente al incremento de los porcentajes que corresponden a los demás niveles, de manera espectacular los estudios de segundo grado. Mientras que en la población no estudiantil no llegan al 10 por 100 los españoles que habían alcanzado los estudios de segundo grado, en 1970 más de la cuarta parte de la población estudiantil cursaba dicho nivel de estudios. Este fenómeno no sólo es propio de los habitantes de la zona urbana, sino que se aplica igualmente a las otras dos. Ha sido la explosión difusora de la enseñanza media a todo el país.

De manera análoga ocurre con los estudios universitarios, aunque naturalmente con un incremento más moderado entre los estudiantes actuales y los antiguos. La enseñanza de tercer grado no universitaria no presenta una tendencia clara, fruto de una concurrencia de factores entre los que no es el menos importante la fijación de los estudios que tienen tal carácter pendiente todavía de una regulación definitiva.

Aunque la situación no es tan favorable en las zonas intermedia y rural, se advierte una franca tendencia hacia el acercamiento entre ellas y la zona urbana, que es reflejo de una aproximación a la igualdad de oportunidades de todos los españoles ante la educación.

Muchas otras características pueden ser objeto de análisis, apoyado en la amplia información numérica que contienen la monografía comentada y las monografías provinciales complementarias. Los estudiosos pueden utilizarla para profundizar en el análisis, siempre interesante, de nuestra estructura demográfica y socioeconómica. 
REVL-1974, núm. 184. BALLESTER ROS, IGNACIO. ALGUNAS CARACTERISTICAS DE LA POBLA... REVL-1974, núm. 184. BALLESTER ROS, IGNACIO. ALGUNAS CARACTERISTICAS DE LA POBLA... 
REVL-1974, núm. 184. BALLESTER ROS, IGNACIO. ALGUNAS CARACTERISTICAS DE LA POBLA...

\section{REVISTA}

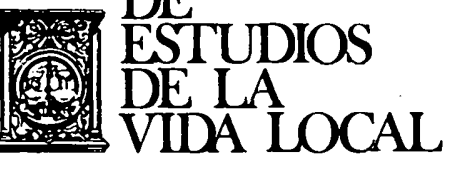

\section{JURISPRUDENCIA}


REVL-1974, núm. 184. BALLESTER ROS, IGNACIO. ALGUNAS CARACTERISTICAS DE LA POBLA... REVL-1974, núm. 184. BALLESTER ROS, IGNACIO. ALGUNAS CARACTERISTICAS DE LA POBLA... 